Die FMH bedankt sich für diesen Beitrag der Schweizerischen Gesellschaft für Kardiologie SGK, welcher wichtige Richtigstellungen zu unserem Grundlagenpapier zu Overuse vornimmt, und in der Diskussion zu dieser Thematik einen weiteren Beitrag leistet. Für die FMH ist es unbestritten, dass die medizinischen Fachgesellschaften für die Fragen ihres Fachgebiets abschliessend zuständig sind. Beiträge wie der hier erscheinende machen einmal mehr sichtbar, dass sich die Ärzteschaft intensiv mit der Indikationsqualität befasst, im Interesse unserer Patientinnen und Patienten.

Christoph Bosshard, Vizepräsident der FMH

\title{
«Overuse» und Qualität
}

\section{Michael J. Zellwegera, Marco Roffi ${ }^{\mathrm{b}}$, Hans Ricklic, François Mach ${ }^{\mathrm{c}}$, Urs Kaufmann ${ }^{\mathrm{d}}$}

a Prof. Dr. med., Vizepräsident der SGK, Präsident der Qualitätskommission; ${ }^{b}$ Prof. Dr. med., Präsident der Arbeitsgruppe interventionelle Kardiologie und akute Koronarsyndrome; ${ }^{c}$ Prof. Dres. med., Vorstandsmitglieder der SGK; ${ }^{d}$ Dr. med., Präsident der SGK

\begin{abstract}
Wohlstand ist die Summe des Überflüssigen, ohne das man nicht mehr auskommen kann. Gustav Knuth (1901-1987)
\end{abstract}

Der Artikel «Overuse - unnötige Behandlungen als Qualitätsproblem» von Gerber et al. [1] bietet einen guten Überblick über die Problematik der medizinischen Überversorgung.

Stete Bemühungen für die Erhaltung einer optimalen Qualität sind unabdingbar. Es ist allerdings nicht nachvollziehbar, warum der Qualitätsfokus so stark auf angeblichen Qualitätsproblemen in der Schweizer Medizin liegt und nicht auf der insgesamt qualitativ guten medizinischen Versorgung, die breit verfügbar ist. Dies ist umso erstaunlicher, da sich die Schweizer Bevölkerung einer der höchsten Lebenserwartungen weltweit erfreut (was allerdings nur zum Teil der medizinischen Versorgung zuzuschreiben ist).

Die Lebenserwartung in der Schweiz stieg bei Männern von 1981 bis 2014 von 72 auf 81 Jahre und bei Frauen von 79 auf 85 Jahre [5, 6]. Die Mortalitätsrate der koronaren Herzkrankheit in der Schweiz (2011) lag tiefer als in Deutschland, Italien, England oder Österreich. Zumin-

\section{Overuse und Qualität}

Unnötige medizinische Prozeduren stellen ein (Qualitäts-)Problem dar. Gerber et al. [1] skizzieren die vielschichtige Problematik der medizinischen Überversorgung gut. Nicht nachvollziehbar ist, dass die Koronarangiographie als Beispiel der medizinischen Überversorgung in der Schweiz angeführt wird; basierend auf Resultaten einer Studie [2], deren Limitationen vielfältig sind. Ein Ländervergleich zeigt zudem, dass die Zahl der Koronarangiographien pro Million Einwohner im Jahr 2009 in der Schweiz (5342) tiefer war als z.B. in Deutschland (6227) oder Österreich (10579) [3]. Die Schweizerische Gesellschaft für Kardiologie arbeitet mit den Richtlinien der European Society of Cardiology (ESC). Diese halten fest, wie Patienten mit Verdacht auf koronare Herzkrankheit abgeklärt werden sollen [4]. dest zum Teil dürfte dieser Mortalitätsrückgang auf die flächendeckende Verfügbarkeit der Reperfusionstherapie mittels Herzkatheter beim Herzinfarkt zurückzuführen sein. Die Mortalität beim Herzinfarkt hat in der Schweiz von 1997 bis 2009 von $12,9 \%$ auf 4,2\% abgenommen [7]. Nach Israel weist die Schweiz mit dieser Mortalitätsrate die zweittiefste Sterberate nach Herzinfarkt in Europa auf [8].

Wie im Artikel von Gerber et al. aufgezeigt, hat die «Overuse»-Diskussion unter anderem mit der Indikationsqualität zu tun, die äusserst komplex und vielschichtig ist; dazu wird erwähnt, dass die Forschung zur Erhebung von «Overuse»-Daten anspruchsvoll sei. Weiter ist der «Overuse» von vielen Faktoren abhängig, nicht zuletzt auch von Patientenerwartungen, Zuweisungspraktiken, Sekuritätsbedürfnissen, medizinischtechnologischen Entwicklungen und Fortschritten. Umso mehr ist nicht nachvollziehbar, dass im Artikel als Beispiel von einem medizinischen "Overuse» in der Schweiz die Koronarangiographie angeführt wird mit der Begründung: «Eine Schweizer Studie belegt, dass bei 34 Prozent der Patienten unangemessene diagnostische Koronarangiographien zur Abklärung von kardiovaskulären Erkrankungen durchgeführt wurden. Leitlinien sehen diese invasive Untersuchung (ein Katheter wird bis ins Herz geschoben) nur vor, wenn vorgängig ein auffälliges Resultat bei einer nicht-invasiven Untersuchung vorliegt. Denn in beinahe 3 Prozent der Fälle treten schwerwiegende Komplikationen auf, wie z.B. ein Schlaganfall.»

Im Artikel von Gerber et al. wird leider nicht auf die vielfältigen Limitationen der zitierten Studie [2] (Appropriateness of diagnostic coronary angiography as a measure of cardiac ischemia testing in non-emergency patients) eingegangen. Erstens bezieht sich die Studie nur auf elektive Patienten und stellt somit bereits eine stark selektionierte Sub-Population der mittels Herz- 
katheter untersuchten Patienten dar. Zweitens hat die genannte Studie lediglich 2,5\% der jährlich angiographierten Patienten evaluiert. Drittens werden die Schlussfolgerungen der Studie durch die präsentierten Versicherungsdaten nicht gestützt. Die Daten erlauben keine Aussage über die "Appropriateness» der durchgeführten Koronarangiographien und der Abklärung der koronaren Herzkrankheit. Warum? Es liegen keine Daten über die Indikation für die Koronarangiographie und über die Beschwerden der Patienten vor, was eine Abschätzung der Vortestwahrscheinlichkeit für eine koronare Herzkrankheit erlauben würde. Die Abschätzung der Vortestwahrscheinlichkeit ist für die Weichenstellung des Abklärungsganges entscheidend (sollen keine weiteren Tests, ein nicht-invasiver Test, oder direkt eine Koronarangiographie erfolgen?).

Folglich dürfte es vermessen sein, zu behaupten, dass $34 \%$ der durchgeführten Prozeduren überflüssig seien. Es stimmt uns nachdenklich, dass die Schweizerische Akademie der Medizinischen Wissenschaften (SAMW) hinter Studien wie der Studie von Chmiel et al. [2] steht und sie sogar als Beispiel für die Förderung einer «nachhaltigen Medizin" propagiert.

Die Schweizerische Gesellschaft für Kardiologie (SGK) arbeitet seit Jahren mit Guidelines/Richtlinien und empfiehlt deren Gebrauch. Meist basieren die Richtlinien auf denen der European Society of Cardiology (ESC) und sind in allen Untergebieten der Kardiologie verfügbar. Beim Entstehungsprozess vieler dieser Richtlinien sind jeweils Schweizer Kardiologen federführend beteiligt.

Betrachtet man die Abklärungsmodalitäten der stabilen koronaren Herzkrankheit in den Richtlinien (die Herzinfarkte stellen ein separates Kapitel dar, wo die Angiographie / katheter-basierte Therapie unbestritten sein dürfte), ist die invasive Koronarangiographie trotz rascher Entwicklung bei den nicht-invasiven Methoden immer noch der diagnostische Goldstandard [4]. Weiter erlaubt sie nach dem diagnostischen Teil mit dem gleichen Gefässzugang in vielen Fällen eine (interventionelle) Therapie. Hinsichtlich Myokardrevaskularisation sind 2014 neu aufgearbeitete europäische Guidelines publiziert worden, nach denen wir uns in der Schweiz richten [9].

Gemäss Guidelines gibt es klar Patientenkollektive, bei denen ein nicht-invasiver Test vor der diagnostischen
Koronarangiographie nicht nötig/angebracht ist [4], zum Beispiel bei Patienten mit hoher Vortestwahrscheinlichkeit für eine koronare Herzkrankheit, Patienten vor Herzklappeninterventionen (chirurgischer oder perkutaner Klappenersatz) und Patienten mit einer Herzinsuffizienz.

Zusätzlich zu den oben festgehaltenen Punkten sei erwähnt, dass die aktuelle Komplikationsrate einer diagnostischen Koronarangiographie weit tiefer liegt als dies im Artikel von Gerber et al. basierend auf dem Artikel von Chmiel et al. [2] und Tages-Anzeiger [10] festgehalten wird. Eine bereits 1989 publizierte Analyse bei über 200000 diagnostisch koronarangiographierten Patienten hielt eine periprozedurale Mortalität von $0,1 \%$ fest [11].

\section{Literatur}

1 Gerber M, Kraft E, Bosshard C. Overuse - unnötige Behandlungen als Qualitätsproblem. Schweiz Ärztezeitung. 97:236-43.

2 Chmiel C, Reich O, Signorell A, Tandjung R, Rosemann T, Senn O. Appropriateness of diagnostic coronary angiography as a measure of cardiac ischemia testing in non-emergency patients - a retrospective cross-sectional analysis. PLoS One. 2015;10:e0117172.

3 Bruckenberger E. Herzbericht 2009.

4 Montalescot G, Sechtem U, Achenbach S, Andreotti F, Arden C, Budaj A, et al. 2013 ESC guidelines on the management of stable coronary artery disease: the Task Force on the management of stable coronary artery disease of the European Society of Cardiology. Eur Heart J. 2013;34:2949-3003.

5 Bundesamt für Statistik. http://wwwbfsadminch/bfs/portal/de/ index/themen/01/06/blank/key/04html

6 Life expectancy. https://wwwciagov/library/publications/ the-world-factbook/rankorder/2102rankhtml

7 Radovanovic D, Erne P. AMIS Plus: Swiss registry of acute coronary syndrome. Heart. 2010;96:917-21.

8 Widimsky P, Wijns W, Fajadet J, de Belder M, Knot J, Aaberge L, et al. Reperfusion therapy for ST elevation acute myocardial infarction in Europe: description of the current situation in 30 countries. Eur Heart J. 2010;31:943-57.

9 Windecker S, Kolh P, Alfonso F, Collet JP, Cremer J, Falk V, et al. 2014 ESC/EACTS Guidelines on myocardial revascularization: The Task Force on Myocardial Revascularization of the European Society of Cardiology (ESC) and the European Association for Cardio-Thoracic Surgery (EACTS). Developed with the special contribution of the European Association of Percutaneous Cardiovascular Interventions (EAPCI). Eur Heart J. 2014;35:2541-619.

10 Straumann F. Ärzte machen vorschnell teure Herzkatheter-Untersuchungen. Tages-Anzeiger. 4.3.2015.

11 Lozner EC, Johnson LW, Johnson S, Krone R, Pichard AD, Vetrovec GW, et al. Coronary arteriography 1984-1987: a report of the Registry of the Society for Cardiac Angiography and Interventions. II. An analysis of 218 deaths related to coronary arteriography. Catheterization and cardiovascular diagnosis. 1989;17:11-4. 